\title{
Uma Estratégia para Institucionalização de Iniciativas para Interdisciplinaridade de IHC aplicada ao Ensino de Programação
}

\author{
Nathalino P. Britto \\ Unifor \\ Fortaleza, Brasil \\ nathalinopacheco@gmail.com
}

\author{
Maria Elizabeth S. Furtado \\ Unifor \\ Fortaleza, Brasil \\ elizabet@unifor.br
}

\author{
Rafaela P. L. Cardoso \\ Unifor \\ Fortaleza, Brasil \\ rafaelaplc@unifor.br
}

\begin{abstract}
IHC's interdisciplinarity teaching solutions generally focus on supporting teachers to plan classes and organise didactic content. Given this context, some studies show that in the algorithms ' disciplines of a computational course there is a logical complexity that requires more attention. In this perspective, we perceive the need to support those involved to implant the interdisciplinarity of IHC in a course that had algorithm discipline. Thus, this article defines and describes a strategy that describes the key elements and activities for such deployment and evaluation. To do so, a case study was carried out to analyze the difficulties in a course for the students of the algorithm discipline to analyze and correct an example with usability error, verified through a heuristic inspection.
\end{abstract}

\section{Author Keywords}

Interdisciplinaridade; IHC; Algoritmos.

\section{ACM Classification Keywords}

K.3.2. Computer and Information Science Education: Computer Science Education, Information Systems Education.

\section{INTRODUÇÃO}

A Sociedade Brasileira de Computação (SBC) formalizou cinco grandes desafios científicos para o período dos dez anos compreendidos entre 2006 e 2016 , aduzindo a questões relacionadas à ausência de interdisciplinaridade, que caracteriza a área da Computação. O Conselho Nacional de Educação (CNE), em 2016, instituiu as diretrizes curriculares nacionais para os cursos de graduação na área da computação e afins, e destacou a formação interdisciplinar para os discentes do curso de Computação. Há vários anos, a SBC vem promovendo eventos para profissionais apresentarem suas práticas docentes, visando discutir as

\footnotetext{
Permission to make digital or hard copies of all or part of this work for personal or classroom use is granted without fee provided that copies are not made or distributed for profit or commercial advantage and that copies bear this notice and the full citation on the first page. Copyrights for components of this work owned by others than the author(s) must be honored. Abstracting with credit is permitted. To copy otherwise, or republish, to post on servers or to redistribute to lists, requires prior specific permission and/or a fee. Copyright 2018 SBC.
}

IHC 2018, Anais Estendidos do XVII Simpósio Brasileiro sobre Fatores Humanos em Sistemas Computacionais

Outubro 22-26, 2018, Belém, Brasil

Workshop sobre Educação em IHC (WEIHC) dificuldades inerentes ao ensino na área ([1]) e sobre as disciplinas de programação, por revelarem o fraco desempenho dos alunos e a evasão. Muitas vezes são utilizados aspectos lúdicos, com o uso de robótica (LEGO) ou de softwares educativos (LOGO), são adotados para auxiliar na aprendizagem dos conceitos, de uma forma prática. Apesar de essas iniciativas facilitarem o aprendizado dos conceitos de lógica de programação, professores e alunos têm interesse maior na geração de código de um software. Consequentemente, as atividades didáticas de avaliação do produto incidem em verificar se o código está funcionando da maneira esperada (sendo testes funcionais), e não em avaliar se o software leva à satisfação do usuário. Esse tipo de avaliação, na perspectiva do usuário, é estudado na disciplina de Interação Humano- Computador (IHC) e difere dos testes funcionais por tratar de aspectos relativos aos fatores humanos.

Diante deste cenário, este artigo foi motivado pela problemática identificada a partir da elaboração e da aplicação do projeto interdisciplinar de IHC em disciplinas introdutórias de programação, quando elas ainda não contam com interface gráfica, no caso de telas prompt. A execução desse projeto com professores de oito turmas trouxe resultados preliminares que motivaram os gestores dos cursos afins à computação a tomarem a decisão de institucionalizar o ensino de IHC. Tal decisão levou às seguintes questões desta pesquisa: Q1) Os docentes envolvidos em um projeto interdisciplinar estariam aptos a ensinarem conteúdos de programação integrados a aspectos de IHC?; Q2) Como o discente percebe tal iniciativa?

Este artigo tem o objetivo de definir e descrever uma estratégia para apoiar os envolvidos (professores e coordenadores de curso) na avaliação da implantação de um projeto interdisciplinar. Um estudo de caso foi feito com a disciplina de IHC aplicada ao Ensino de Programação em diversos cursos afins à computação. Assim, questionários e entrevistas foram aplicados para investigar a percepção da utilidade para os discentes envolvidos e dificuldades dos docentes, e avaliar se existe alinhamento do objetivo do projeto interdisciplinar de IHC com a disciplina-alvo (Programação). Os resultados mostraram que um processo interdisciplinar vai além de relacionar duas disciplinas e gerar um novo conhecimento ou apenas relacioná-las, sendo notório que deve existir alinhamento com vários elementos 
(disciplina (ementa), perfil do docente e discente) para que o objetivo do projeto interdisciplinar seja alcançado e em conjunto melhore o entendimento de conceitos muitas vezes abstrato de programação para os discentes.

A estrutura deste artigo é a seguinte: após uma conceituação sobre usabilidade e experiência do usuário no contexto educativo, é relatado uma prática de um projeto interdisciplinar, sendo posteriormente, descrita a estratégia para avaliação de um projeto interdisciplinar e o estudo de caso, com os resultados alcançados e trabalhos relacionados, antes da conclusão.

\section{USABILIDADE E EXPERIÊNCIA DO USUÁRIO NO CONTEXTO EDUCATIVO}

Os pesquisadores deste artigo estão em um grupo que agrega profissionais que estudam aspectos relativos à interação do ser humano com um artefato computacional, visando a usabilidade de tal artefato, dentre outras características, que possam medir a qualidade das experiências do usuário-alvo. Por conta deste contexto e da limitação de espaço, ressaltase somente que a "Usabilidade" ([5]) é um conceito restrito se comparado ao conceito de Experiência do Usuário (EU) ([6]), que aborda uma questão maior, verificando se um sistema/produto/serviço é bom o bastante para proporcionar satisfação aos usuários durante o seu uso normal e antecipado do sistema. Sem querer fazer uma análise aprofundada, chamaremos simplesmente de usabilidade, sabendo que um pode afetar o outro. Por exemplo, no contexto de ensino, as abordagens de ensino não devem focar apenas nas competências dos alunos (p.e., dos conceitos e princípios de usabilidade de um software) e suas habilidades (p.e., realizar os procedimentos para aplicar os princípios), mas nos valores humanos agregados pelo aprendizado, isto é, na formação de um aluno consciente de sua responsabilidade social, para construir um software útil para os usuários-alvo. Assim, pode-se dizer que, em uma disciplina de programação para resolver um dado problema, é necessário que o aluno compreenda a lógica que ele vai solucionar, mapeando as soluções em passos sequenciais (algoritmo), mas, acima de tudo, compreenda os fatores humanos a serem considerados nas EU dos usuários.

Ainda neste contexto de ensino, as principais estratégias de integração disciplinar são: multidisciplinaridade, pluridisciplinaridade e interdisciplinaridade. Adotou-se a interdisciplinaridade neste trabalho de pesquisa, pois, enquanto as duas primeiras requerem apenas uma consulta nas disciplinas, a última exige ir além, como relacioná-las. Conforme [3], interdisciplinaridade é utilizada para definir "o nível em que a interação entre várias disciplinas ou setores heterogêneos de uma mesma ciência conduz a interações reais, a uma certa reciprocidade no intercâmbio, levando a um enriquecimento mútuo".

\section{CONTEXTUALIZAÇÃO: APLICAÇÃO DE UM PROJETO INTERDISCIPLINAR}

Esta seção objetiva descrever uma iniciativa de um projeto interdisciplinar, tomando como base as disciplinas de IHC e de Algoritmos, que motivou a elaboração da estratégia proposta.

O projeto iniciou e foi aplicado em 2014.2 na Unifor (Universidade de Fortaleza) pelos seguintes integrantes da equipe: os autores deste artigo (sendo um especialista em IHC, uma pedagoga e um programador), o coordenador do curso de ciência da computação e um professor representante da disciplina Algoritmos. O objetivo foi preparar, executar e disponibilizar os recursos necessários aos professores para conduzirem seus alunos a programarem dando atenção para a usabilidade da solução, desde o início da construção de um programa, e atingindo os três saberes. Ficou definido pela equipe que, no decorrer de duas aulas, os alunos deveriam i) compreender o conceito de IHC, para ter o foco na usabilidade da aplicação; ii) avaliar uma tela interativa de cadastro de usuário, aplicando heurísticas de usabilidade para identificar erros de usabilidade; e iii) perceber a necessidade do uso de heurísticas nas estruturas de controle durante a construção de programas na linguagem " $C$ " (linguagem utilizada na disciplina). Como ponto de associação aos conceitos de programação da disciplina de algoritmos, as heurísticas identificadas foram: visibilidade, tratamento de erros, liberdade e controle do diálogo e consistência e padronização. A Tabela 1 ilustra uma heurística de IHC associada ao conceito de programação da disciplina de algoritmos.

\begin{tabular}{|c|c|}
\hline Heurística & $\begin{array}{c}\text { Heurística associada ao conceito de } \\
\text { programação }\end{array}$ \\
\hline Visibilidade & $\begin{array}{l}\text { Trata-se de fornecer feedback } \\
\text { adequado para as ações do usuário e } \\
\text { para o estado do programa. Uma das } \\
\text { possibilidades que é utilizada para } \\
\text { fornecer feedback é utilizar a função } \\
\text { "printf”. Exemplo: Quando é } \\
\text { solicitado o sexo do usuário, não } \\
\text { podemos nos esquecer de fornecer } \\
\text { mensagens claras, indicando } \\
\text { (tipo/tamanho faixa) os valores } \\
\text { esperados para o campo: } \\
\text { printf ("InQual o seu sexo?"); } \\
\text { printf ("In[ F ] - Feminino.”); } \\
\text { printf ("In[ M ] - Masculino.”); } \\
\text { printf (“InMeu sexo é:"”); }\end{array}$ \\
\hline
\end{tabular}

Tabela 1. Heurística associada ao conceito de programação.

A metodologia pedagógica adotada foi a Sequência Fedathi ([2]), que ajudou a equipe a identificar os conceitos de IHC e definir como eles deveriam ser produzidos e integrados com os conceitos da disciplina Algoritmo. Essa metodologia privilegia a apresentação de situações- problema aos alunos, que se debruçam e refletem sobre possíveis respostas e constroem um conhecimento, enquanto o professor é um mediador do processo de ensino- aprendizagem ([2]). Ficou definido pela equipe que os docentes iriam receber um kit, 
contendo o plano de aula, o programa exemplo, o checklist para avaliação da usabilidade do programa, slides sobre a associação entre os conceitos das disciplinas e duas versões do programa: com erros de usabilidade e sem erros de usabilidade.

Após a elaboração do projeto, houve o treinamento dos professores e a realização das aulas para os alunos. É nesse contexto que as dificuldades dos professores foram apuradas para a avaliação do projeto.

A equipe treinou 8 dos 15 professores da disciplina de Algoritmos que na época eram responsáveis em lecionar. $\mathrm{O}$ treinamento ocorreu em três momentos, com duração de quase três horas (equivalente a 2 aulas). No primeiro momento, após uma revisão dos conceitos de algoritmos que seriam usados, a tela de cadastro foi apresentada pelo professor de algoritmos e os oito professores (na posição de alunos) foram motivados a identificar e apontar os erros de usabilidade. O professor de algoritmos foi anotando no quadro os erros identificados, considerando as repetições e divergências de opinião. $\mathrm{O}$ especialista de IHC percebeu que muitos dos erros anotados não eram de usabilidade e sim de funcionalidade, e esclareceu que um programa só pode ser avaliado do ponto de vista da satisfação do usuário quando não existirem mais falhas que impeçam o seu funcionamento correto. Após o entendimento dos conceitos e da apresentação de heurísticas de usabilidade, iniciou-se o segundo momento, os professores-aluno, de forma individual, avaliaram a tela, passando o checklist fornecido. No terceiro momento, os dois professores (IHC e Algoritmos) sistematizaram as possíveis respostas, usando a lista de problemas identificados. Para tanto, os professoresaluno apresentaram suas respostas acerca do exercício e os professores que ministraram a oficina chamavam a atenção de que a inspeção da usabilidade por meio do checklist é um processo de avaliação subjetiva. Para finalizar, as soluções associativas de IHC com os comandos em $\mathrm{C}$ foram apresentadas para os professores-aluno, motivando-os a tratarem a usabilidade desde o estágio inicial de desenvolvimento de um programa. Os comentários dos professores foram positivos. Este conceito de IHC estudado passou a fazer parte das ementas das disciplinas de Algoritmos em todos os cursos na qual é disponibilizado, devido ao sucesso do projeto interdisciplinar. Apesar desse avanço, a equipe sabia que as Q1 e Q2 precisavam ser investigadas.

\section{UMA ESTRATÉGIA PARA AVALIAÇÃO DA INTERDISCIPLINARIDADE}

A estratégia proposta é composta de elementos (ver Figura1) sobre a disciplina-alvo (a disciplina que terá seu conteúdo integrado ao conteúdo de IHC num certo projeto), o docente e o discente. A ementa da disciplina-alvo se refere aos conteúdos que serão abordados no projeto. É esperado que o docente da disciplina-alvo tenha um conhecimento prévio/domínio e/ou seja especialista na área, bem como tenha sido treinado nos conceitos de IHC (como descrito na seção anterior). Quanto ao discente, é avaliar o entendimento e a importância do projeto interdisciplinar.

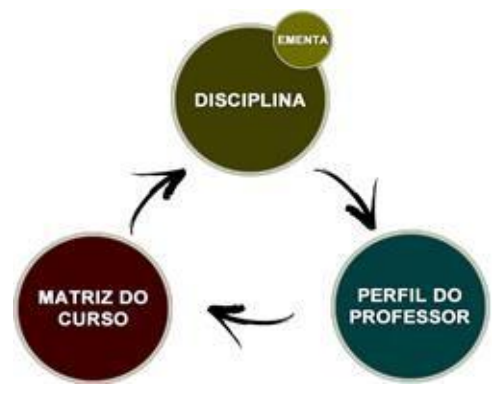

Figura 1. Elementos.

A estratégia conta também com uma metodologia de avaliação do projeto em questão. A metodologia de avaliação é composta de três principais atividades: i) uma avaliação inicial para diagnóstico do discente (foi proposto um questionário inicial a ser aplicado com todos os discentes); ii) acompanhamento do ensino da unidade interdisciplinar (no caso, da aplicação de um projeto interdisciplinar - como o da seção anterior), e; iii) uma avaliação final (foi feita uma entrevista com os envolvidos), após o conteúdo da disciplina. Nessa metodologia, os elementos também devem ser avaliados por meio de uma análise do projeto de ensino da disciplina, currículo lattes, website da universidade e diálogos com o coordenador da disciplina estudada. A próxima seção apresenta cada uma dessas atividades, descritas para um estudo de caso realizado.

\section{ESTUDO DE CASO: APLICAÇÃO DA ESTRATÉGIA}

$\mathrm{Na}$ Unifor, ocorreu o estudo de caso na disciplina de Programação chamada: Algoritmos e Programação de Computadores (APC), com o professor Mateus Mosca Viana, que participou do treinamento descrito na seção: "Contextualização: Aplicação de um projeto interdisciplinar". O projeto foi realizado em três aulas consecutivas por esse professor e ocorreu de acordo com o cronograma da ementa seguida pelo docente, pois era preciso aguardar a chegada da unidade $\mathrm{V}$, denominada Interface Humano-Computador, sendo uma herança do projeto interdisciplinar contextualizado nesse artigo. A unidade $\mathrm{V}$ é dividida em três subunidades, nas quais a subunidade 05.01 se refere às heurísticas de IHC associadas a conceitos de programação; subunidade 05.02 refere-se à validação de uma interface e a última subunidade 05.03 trata-se de uma atividade prática para os discentes exercitarem os conceitos integrados. Assim, durante o mês de outubro de 2017.2, acompanhamos sua turma de 50 alunos. A metodologia de avaliação mencionada na seção anterior foi realizada para esse estudo de caso. Usou-se o Formulários Google para aplicação do questionário inicial, um dia antes do início da unidade $\mathrm{V}$ e, em seguida, foi realizada uma entrevista, após o término da referida unidade $\mathrm{V}$.

Análise dos Resultados quanto ao Perfil dos Docentes (Q1) 
Conversando com o coordenador da disciplina de APC e fazendo a análise das matrizes curriculares dos cursos, foi averiguado que essa disciplina é comum a 9 cursos de engenharias: Ambiental e sanitária; civil; computação; controle e automação; elétrica; eletrônica; mecânica; produção e telecomunicações. Nos cursos mencionados, é comum a disciplina estar no primeiro período, exceto nos cursos de engenharia ambiental e sanitária (quarto período) e de engenharia de produção (segundo período). Sobre os objetivos dessa disciplina, conforme a ementa são: ter fundamentos da computação; estudar métodos para a resolução de problemas utilizando raciocínio lógicos; tipos e estruturas de dados; estruturas de controle; abstração e manuseio de dados. Atualmente, a disciplina dispõe de 7 professores responsáveis em ensiná-la. Explorando os currículos lattes desses professores, através do próprio website da universidade nas seções: "Formação Acadêmica/Titulação", "Formação complementar" e "Atuação profissional" dos docentes, foi possível constatar que os professores que assumem a disciplina-alvo têm um perfil que convergem para área da tecnologia, ou seja, possuem graduação, especialização, mestrado ou doutorado na área da tecnologia, mas não foi encontrado nenhum docente com especialização ou atuação profissional com ênfase na área de IHC. A maioria dos currículos lattes dos docentes estava atualizado no ano de 2017.2.

\section{Análise dos Resultados quanto à Percepção do Discente(Q2)}

A aplicação dessa metodologia visou verificar a Q2 desta pesquisa, mas antes de ocorrer os acompanhamentos, foi necessário um diálogo com o professor para coletar o seu ponto de vista sobre o projeto interdisciplinar, sendo que o mesmo já tinha um embasamento de mais de 2 anos. O professor narrou que achava fundamental a proposta, pois o projeto favorece aos alunos o entendimento da lógica para programação.

Analisando o questionário inicial aplicado com 23 alunos presentes no dia da aula, verificamos que a turma era heterogênea. Os discentes eram de 5 cursos diferentes, sendo que o curso de engenharia civil é predominante com 10 discentes, engenharia mecânica (5 alunos), engenharia elétrica (3 alunos), produção (3 alunos) e engenharia de controle e automação com 2 alunos.

Houve uma pergunta subjetiva sobre o que o discente esperava da disciplina de APC para a sua vida profissional. Os objetivos de aprendizagem principais da disciplina são aprender a "lógica"; "programar" e "solucionar problemas"; então, procuramos essas palavras nas respostas dos discentes. Foi constatado que mais de $74 \%$ dos discentes utilizaram essas palavras, trazendo evidências para esta pesquisa, de que os discentes tinham expectativas de entender a lógica de programação, programar e/ou solucionar problemas na sua profissão, logo este resultado pode ter sido verificado, porque, no decorrer do processo de ensino da unidade $\mathrm{V}$, observou-se que o professor teve segurança ao abordar os conceitos.

Em outro questionamento, objetivou-se verificar se o discente, ao programar uma determinada atividade prática interativa rotineira da disciplina, chegava a pensar no usuário que utilizaria a aplicação. Cerca de $87 \%$ pensaram de alguma forma no usuário e apenas $13 \%$ não pensaram no usuário que utilizaria a aplicação interativa.

A penúltima indagação investiga a percepção deles sobre a utilidade da usabilidade. Identificamos que $96 \%$ dos discentes consideram importante estudar usabilidade na disciplina de Algoritmo. É relevante salientar que essa indagação foi fundamentada com conceitos de diversos autores sobre "o que é usabilidade", logo é interpretado que os discentes não tinham conhecimento sobre o conceito da palavra.

Finalmente, foi analisada a última pergunta: "o discente conhecia princípios para projeto de programas (aplicações interativas), como: visibilidade, tratamento de erros, liberdade e controle do diálogo pelo usuário e consistência e padronização?". Foram obtidas as seguintes respostas: 74\% não conheciam nenhum desses princípios e $26 \%$ conheciam alguns, sendo estes não identificados. Não foi realizada nenhuma introdução em cada heurística. Esses dados trouxeram evidências de que os alunos não tinham um conhecimento prévio sobre as heurísticas, antes de o professor lecioná-las.

Concluindo aplicação do questionário inicial, começou a unidade $\mathrm{V}$ e os discentes participaram ativamente da aula. Eles, inclusive em alguns momentos antes do docente, apresentaram como se resolveria problemas de heurísticas; já citavam conceitos da disciplina de APC, apresentando o entendimento da associação (ver exemplo na Tabela 1).

A entrevista, última etapa da estratégia, ocorreu com os 23 alunos participantes. A seguinte pergunta foi feita: "Como poderíamos resolver problemas de usabilidade, considerando as heurísticas de IHC no momento da aplicação dos conceitos da disciplina de APC? Então foi solicitado aos entrevistados que explicassem, verbalmente, como se resolveria os erros de usabilidade de uma tela de login, utilizando as heurísticas de IHC associadas aos conceitos de APC (ver um exemplo na Tabela 1) fundamentadas pela linguagem de programação "C", que é a linguagem utilizada na disciplina. É necessário enfatizar que os alunos receberam uma imagem (ver Figura 2) da tela de login (Essa tela não foi trabalhada em sala de aula pelo professor) e uma tabela contendo as seguintes heurísticas: visibilidade; tratamento de erros; liberdade e controle do diálogo e consistência e padronização. Nesse instante, os discentes já tinham um conhecimento conceitual dessas heurísticas, logo tinham trabalhado em sala de aula. 


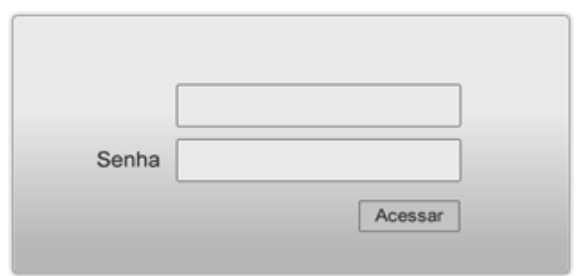

Figura 2. Tela de login com erro de usabilidade.

$\mathrm{Na}$ primeira heurística visibilidade, todos os discentes responderam que faltava um título de identificação para a tela de login e o campo para dar entrada no login do usuário precisava de uma etiqueta, conforme havia no campo de senha e, para resolver esses problemas de visibilidade, falaram da função "printf" da linguagem "C". Sobre a heurística tratamento de erros, os discentes falaram da importância da verificação dos tipos de dados que são inseridos pelo usuário, para isso, alguns disseram que adotariam os controles de fluxo "if" (se), "while" (enquanto), "for" (para), tratando assim os tipos de dados e verificando a coerência do login e senha cadastrados. Quanto à heurística liberdade e controle do diálogo pelo usuário, 5 discentes não souberam resolver esse problema e os demais relataram não haver a necessidade de se preocupar com a heurística, justamente pelo usuário ter a necessidade de responder os dois campos para ter acesso a próxima etapa do sistema, mas esqueceram de pensar que o usuário poderia chegar naquela tela de login por engano. É essencial que existam opções para retorno ou sair/finalizar na tela de login. A última heurística, consistência e padronização, todos consideraram que o programador deveria seguir uma padronização nas mensagens de erros e, se possível, com uma opção para o usuário refazer o processo.

\section{TRABALHOS RELACIONADOS}

Iniciou-se a coleta de trabalhos relacionados, visando apresentar respostas para as perguntas: "Em quais disciplinas ocorreram interdisciplinaridade com IHC?", "Quais os pontos relevantes sobre um projeto interdisciplinar?" e "Qual o comportamento requerido do docente e do discente para a interdisciplinaridade implantada?" e outros fatos peculiares que pudessem contribuir para o nosso projeto. Sobre a busca, são utilizadas as palavras-chave: IHC e Programação e Interdisciplinaridade de IHC com programação nas três das principais bases digitais de trabalhos científicos: Google Acadêmico, Scielo Brasil e Periódicos Capes/MEC. Houve também uma busca manual exaustiva em alguns anais do evento WEIHC (Workshop sobre Ensino de IHC), por considerar que o WEIHC é berço para vários pesquisadores expor os seus trabalhos na área de ensino de IHC. Dentre os 8 trabalhos identificados nas bases, de acordo com as palavras-chave, destacaram-se 3 pelos traços lineares com o nosso tema, descritos em termos de temas. Os temas que aqui se definem não são o título do artigo. Eles foram definidos para apresentar, de forma clara, que disciplinas fazem interdisciplinaridade (Primeira indagação coletada no artigo).
- Interdisciplinaridade do Ensino de IHC nas disciplinas de Modelagem e Projeto de Banco de Dados e Projeto de Sistemas. Os autores [7] relatam um projeto interdisciplinar com o conteúdo de três disciplinas: 1) Modelagem e Projeto de Banco de Dados; 2) Projeto de Sistemas; e 3) Projeto de Interface Homem-Computador. Tal experiência foi executada no $5^{\circ}$ período do curso de Ciências da Computação. $\mathrm{O}$ projeto interdisciplinar vem ocorrendo de forma que os conteúdos dessas disciplinas sejam aplicados em um projeto de cunho teórico-prático. $\mathrm{O}$ projeto interdisciplinar entre as três disciplinas tem como foco integrar as visões sobre os dados, funcionalidades e interface de um sistema. Sobre as implicações da interdisciplinaridade para os envolvidos (alunos), os autores criaram um questionário com duas perguntas para que comentassem sobre pontos positivos e negativos do projeto. Como pontos positivos, os alunos narraram: "desafiante, abordagem prática"; "visão ampla do programa a ser criado" e "o projeto deu uma visão de como é o mercado de trabalho e a compreensão de que é exigido de um bom profissional o desenvolvimento de um produto com qualidade" e os pontos negativos foram: "que precisavam de mais tempo para tirar dúvidas sobre o projeto durante as aulas" e "apenas uma aula de IHC por semana";

- Interdisciplinaridade do Ensino de IHC na disciplina de Programação. O trabalho de [8] apresenta uma experiência de integração curricular que foi pautada na articulação conceitual de duas disciplinas de referência: IHC e Programação (Plataforma NET I - com ênfase em recursos e controles básicos da Linguagem C\#). Uma questão a ser levada em consideração nesse artigo é que houve relação com IHC por existir um tópico (design de interfaces do usuário) específico na disciplina, que está intimamente relacionado aos estudos de IHC. Para o autor, o docente tem que ter uma experiência na área de IHC para poder utilizar a didática que é dividida em dez horas-aula. O autor aplicou avaliações durante a intervenção didática e, ao final da disciplina, como resultado nas atividades tanto individuais como coletivas, o autor observou que os estudantes fizeram projetos de interação e apresentaram softwares finais (Programados em C\#) que representavam fielmente o design de interação. Além dessas avaliações, houve relatos de alguns alunos ao final da aula e em redes sociais, favorecendo as inferências sobre a integração curricular; e.

- Interdisciplinaridade de IHC com as disciplinas de Análise e Projeto de Sistemas I e Programação em Ambiente Web. Os autores [4] relatam a experiência da prática de um projeto interdisciplinar que foi desenvolvido em uma turma de alunos do $5^{\circ}$ semestre do curso de Bacharelado em Sistemas de Informação. As disciplinas envolvidas nesse projeto são: IHC; Análise e Projeto de Sistemas I e Programação em Ambiente Web. Segundo os autores, o objetivo da proposta é que o aluno consiga, além de adquirir os conhecimentos de cada disciplina, 
compreender a relação e integração dos assuntos inerentes às três. Sobre o papel de cada disciplina, os autores asseveram que alguns exercícios foram estabelecidos de forma interdisciplinar, a exemplo das técnicas de levantamento de requisitos e testes, em que tanto a disciplina de Análise e Projeto de Sistemas I, como a de IHC estabeleceram técnicas, métodos e ferramentas. Houve um momento de diálogo entre os professores e alunos, em que foram relatadas suas experiências durante a execução do projeto. Após os relatos dos alunos, foram evidenciadas algumas dificuldades relacionadas à falta de conhecimento e às experiências sobre a metodologia de projeto interdisciplinar. Nenhum aluno conhecia tal método. Já o ponto positivo relatado foi a visão que eles tiveram sobre a relação e a integração das disciplinas envolvidas.

\section{CONCLUSÃO}

Apesar de a área de Computação ser relativamente nova, em comparação a outras áreas que já possuem uma vasta base de conhecimento, é notória a pouca quantidade de pesquisas que estudam ou executam a interdisciplinaridade da área de IHC com disciplinas de programação no Brasil. Assim, este artigo descreveu, sucintamente, a aplicação de um projeto para planejar um plano de aula para interdisciplinaridade de IHC em disciplinas de algoritmos, quando a programação ainda faz uso de tão poucos recursos gráficos (Prompt).

Realizou-se um estudo de caso, seguindo a estratégia proposta para avaliar um projeto em implantação para a interdisciplinaridade de IHC em disciplinas de algoritmos. Assim, aplicou-se uma estratégia no estudo de caso a partir da análise de três elementos principais: i) Em alguns cursos, a matriz curricular em questão não possuía disciplinas de IHC. Portanto, uma das formas de os discentes conhecerem alguns conteúdos de IHC é por meio da interdisciplinaridade. É importante realçar que IHC participou da interdisciplinaridade por ter o seguinte foco: o estudo da usabilidade na aplicação; ii) $\mathrm{Na}$ ementa do curso, foi observado o alinhamento do projeto interdisciplinar apresentado na seção: "Contextualização: Aplicação de um projeto interdisciplinar" com o objetivo da disciplina em si. $\mathrm{O}$ projeto foi idealizado para fazer interdisciplinaridade de conceitos de algoritmos com as heurísticas de IHC, sendo que as heurísticas visam nortear para possíveis resoluções de problemas de usabilidade, e os conceitos utilizam a lógica para solucionar alguns desses problemas; e iii) Com relação aos docentes, mesmo sendo oriundos da área de tecnologia, comprovamos, via currículo lattes, que nenhum dos docentes possuía alguma especialização ou atuação profissional com ênfase na área de IHC. Desse modo, torna-se lógico inferir que os docentes que venham a lecionar a disciplina de algoritmos e cheguem no tópico que abranja a abordagem interdisciplinar, possivelmente tenham dúvidas, sendo sugerido um apoio de docentes da área de IHC.

Sobre as pesquisas com os discentes, foi comprovado, através de dados, que o discente, em certas atividades rotineiras da disciplina, ao desenvolver uma determinada atividade prática interativa, chega a pensar de alguma forma no usuário que utilizaria aquela aplicação. Mas se não houver subsídios na própria disciplina para atendê-lo, então, continuará com a dúvida se deve ou não pensar no usuário que vai utilizar a aplicação interativo.

Como trabalhos futuros, pretende-se avaliar como os outros docentes, que foram treinados, poderão contribuir com a evolução da abordagem; a percepção dos professores após terem ministrado suas aulas, quanto à satisfação dos alunos para os seguintes itens: i) os conceitos de IHC foram simples e fáceis de aprender? ii) o material didático do kit disponibilizado foi suficiente? Além disso, seria interessante verificar em que condições as organizações educativas estariam dispostas a tratar a interdisciplinaridade em seus processos de ensino-aprendizagem.

\section{REFERÊNCIAS}

1. Bim, S. A., Silveira, M. S. e Prates, R. O. Ensino de IHC: compartilhando as experiências docentes no contexto brasileiro. In: Proceedingsofthe11th Brazilian Symposiumon Human Factors in Computing Systems (IHC 2012), 195-198,2012.

2. Cardoso, R. P. L., Furtado, M. E. S., Borges Neto, H. e Silveira, C. Formação de telespectadores para TVD Interativa apoiada na Sequência Fedathi e Aplicada para o Desenvolvimento de Programas Jornalísticos. Anais do Simpósio Brasileiro de Informática na Educação, 24, 1,2013.

3. Chaves, Mario M. Complexidade e transdisciplinaridade: Uma abordagem multidimensional do Setor Saúde.1998. Disponível em: <http://ibiblio.org/cedros/complexi.htm>. Acesso em: 28 mar. 2018.

4. De Souza, Patricia Cristiane e Freiberger, Evandro C. A Prática do Projeto Interdisciplinar como Suporte ao Ensino de IHC. II WEIHC, 2011.

5. ISO 9241-11. Geneve: InternationalOrganization For Standardization.1998.

6. ISO DIS 9241-210:2008. Ergonomicsofhuman system interaction -Part 210: Human -centred design for interactive systems (formerlyknownas 13407). InternationalStandardizationOrganization (ISO). Switzerland.2008.

7. Marques, Anna Beatriz dos S. e Melo, Áurea Hiléia da Silva. Relato de experiência no ensinode IHC em nível de graduação através de uma abordagem interdisciplinar. In: Anais do VI Workshop sobre Ensino de IHC-XIV.2015.

8. Matos, Ecivaldo de Souza. Integração curricular por meio da prática de ensino interdisciplinarem IHC. In: WEIHC. 2013. p.25-30. 